'Sección de Neumología Intervencional, Clínica AlemanaUniversidad del Desarrollo, Santiago. Chile

${ }^{2}$ Facultad de Medicina, Pontificia Universidad Católica de Chile, Santiago. Chile.

'Departamento de Enfermedades Respiratorias, Clínica AlemanaUniversidad del Desarrollo, Santiago, Chile. Interno de Medicina. ${ }^{\text {b}}$ Residente de Medicina Interna.

*Todos los autores autorizaron la versión final del borrador y declaran no tener conflicto de interes ni financiamiento por el manuscrito

Recibido el 11 de agosto de 2015, aceptado el 21 de noviembre de 2015

Correspondencia a Dr. Fernandez-Bussy Manquehue Norte 1410,

Vitacura, Santiago. Chile sfernandezbussy@alemana.cl

\section{Lavado broncoalveolar y derrame pleural con hipereosinofilia. Caso clínico y revisión de la literatura}

\author{
SEBASTIÁN FERNÁNDEZ-BUSSY ${ }^{1}$, FELIPE CAMPOS ${ }^{1}$, \\ ISABEL OGUETA ${ }^{2, \mathrm{a}}$, GONZALO LABARCA ${ }^{2, \mathrm{~b}}$, HERNÁN CABELLO $^{3}$
}

\section{Chronic eosinophilic pneumonia. Report of one case}

Chronic eosinophilic pneumonia (CEP) is uncommon and predominantly seen in women. More than 6\% of eosinophils in peripheral blood and more than $25 \%$ in bronchoalveolar lavage are diagnostic criteria. Secondary causes of hypereosinophilic pneumonia must be ruled out. We report a 72-year-old non-smoker man presenting in the emergency room with a history of cough, fever, and moderate dyspnea. He was not taking any medication. A chest-X ray showed a left lower lobe (LLL) consolidation, and was started on broad-spectrum antibiotics with a presumptive diagnosis of pneumonia. There was no improvement after therapy. A chest CT scan showed increased LLL consolidation and new left upper lobe ground glass opacities as well as a moderate left pleural effusion. Flexible bronchoscopy was performed and bronchoalveolar lavage showed 95\% eosinophils, and had negative cultures. No parasites were identified. Transbronchial biopsies demonstrated eosinophil accumulation in alveoli and interstitium and pleural fluid was composed by 85\% eosinophils. With the diagnosis of CEP, systemic corticosteroids were used with favorable clinical and radiological response.

(Rev Med Chile 2016; 144: 262-266)

Key words: Eosinophilia; Lung Diseases; Pneumonia; Pulmonary Eosinophilia.
L a neumonía eosinofílica crónica (NEC) es una patología de baja prevalencia, vista sobre todo en mujeres de aproximadamente la quinta década de vida, pudiendo encontrarse historia de asma o atopia en 50\% de los pacientes. Inicialmente fue incluida en un grupo de diversas enfermedades que se caracterizaban por compromiso pulmonar y eosinofilia, sin embargo, en 1969, Carrington et al. la definieron como una entidad propia e independiente ${ }^{1}$. Reportamos el caso de un hombre adulto mayor que presentó un cuadro de fiebre, tos crónica y disnea moderada, con una revisión de la literatura de esta entidad tan poco frecuente.

\section{Caso clínico}

Se comunica el caso de un hombre de 72 años de edad, no fumador, evaluado en la sala de urgencias por un cuadro de 15 días de evolución de tos seca, fiebre y disnea moderada. Sin antecedentes de asma ni rinitis alérgica, tampoco refiere uso de medicamentos; familia niega uso de antiinflamatorios no esteroidales, antibioterapia previa y cocaína u otras drogas de abuso. La hemodinamia y oximetría se encontraban en rango normal. Al examen segmentario se encontraron crépitos en base pulmonar izquierda, sin sibilancias ni otros ruidos agregados; sin hipocratismo digital ni 
otros signos sugerentes de patología pulmonar previa y el resto del examen físico fue normal. La radiografía de tórax mostró foco de consolidación en el lóbulo inferior izquierdo (LII). Dentro de sus exámenes de ingreso destacaba leucocitosis de $13.200 \mathrm{cel} / \mathrm{dL}$, velocidad de eritrosedimentación (VHS) de $65 \mathrm{~mm}$, proteína $\mathrm{C}$ reactiva de 15 (valor normal $<5$ ), perfil bioquímico normal. Con estos hallazgos se decidió hospitalización y se dio inicio a antibioterapia con ceftriaxona $2 \mathrm{~g} /$ día ev y levofloxacino $500 \mathrm{mg}$ al día vo. Posterior a una semana de tratamiento, no hubo mejoría clínica. Estudios de laboratorio con hemograma mostraron hematocrito 38\%, hemoglobina $12 \mathrm{~g} /$ dL, VCM 89, leucocitos $12.500 \mathrm{cel} / \mathrm{mm}^{3}$, eosinófilos $16,9 \%$, plaquetas $234,000 \mathrm{cel} / \mathrm{mm}^{3}$,VHS 35 mm, PCR 15 (límite < 5) Ig E 128,2 UI/ml. Tomografía computarizada de tórax (TAC) mostró una mayor consolidación de LII y una nueva en lóbulo superior izquierdo (LSI), asociado a opacidades en vidrio esmerilado, así como un derrame pleural izquierdo moderado (Figura 1). Se realizó broncoscopia flexible con lavado broncoalveolar (LBA) que mostró un total de 2,5 × $10^{7}$ células, con 95\% de eosinófilos (Figura 2); los cultivos, tanto en medios corrientes como micobacterias, fueron negativos y los niveles de galactomanano fueron normales. La biopsia transbronquial mostró numerosos granulocitos eosinófilos en alvéolos e intersticio, asociado a cierto grado de fibrosis intersticial. El análisis del líquido pleural arrojó 1,2 x $10^{3}$ células, con $85 \%$ de eosinófilos, proteínas 4,1 $\mathrm{mg} / \mathrm{dL}$, glucosa $75 \mathrm{mg} / \mathrm{dL}$, lactato deshidrogenasa 232 y pH 7,37 (Figura 3).

Dentro del estudio complementario, se solicitaron anticuerpo antinucleares (ANA), anti DNA, anti-citoplasma de neutrófilos (ANCA), todos negativos. El estudio para virus de inmunodeficiencia humana (VIH) fue negativo y se descarto la presencia de infección por parásitos (Ascaris lumbricoide, Trichinella, Fasciola, Schis-

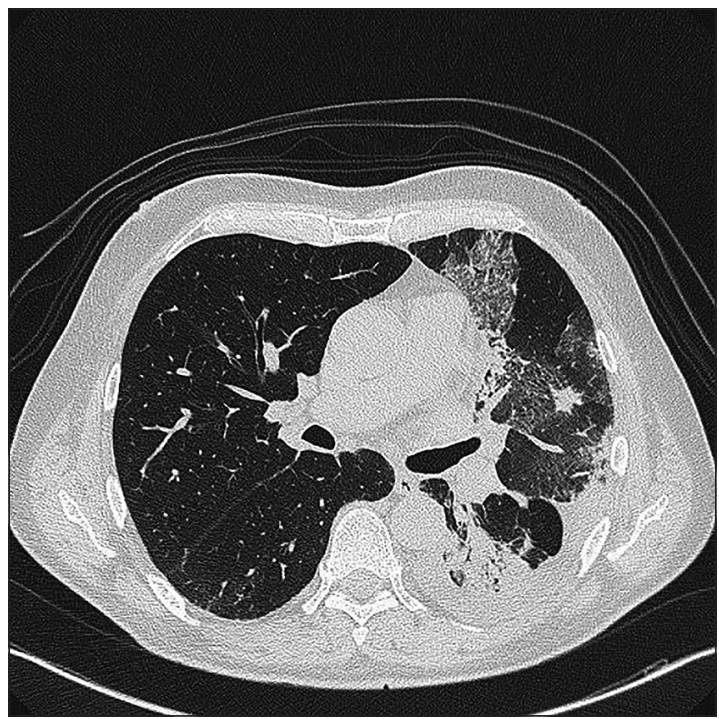

Figura 1. TAC de tórax que muestra opacidades en vidrio esmerilado en lóbulo superior izquierdo, y consolidación en base inferior izquierda asociado a derrame pleural izquierdo.

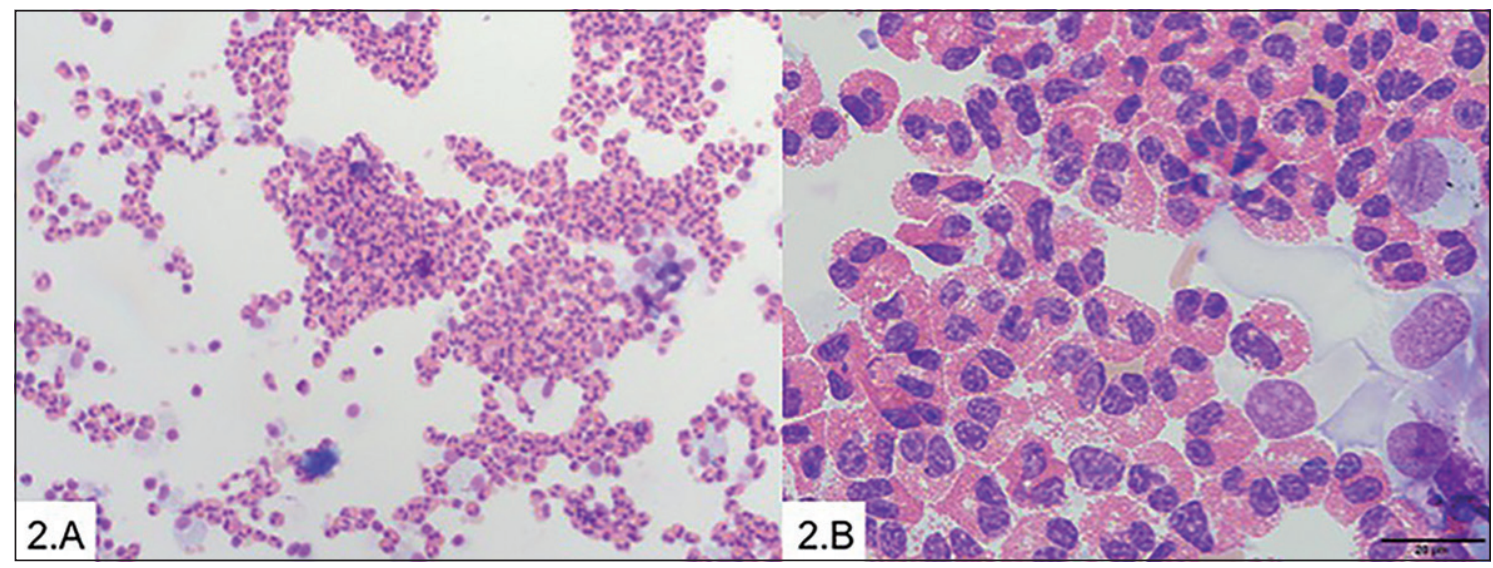

Figura 2. Citología de lavado broncoalveolar que muestra abundantes eosinófilos (células nucleadas en naranjo); macrófagos (células grandes, violeta) y linfocitos (células pequeñas de color violeta). 


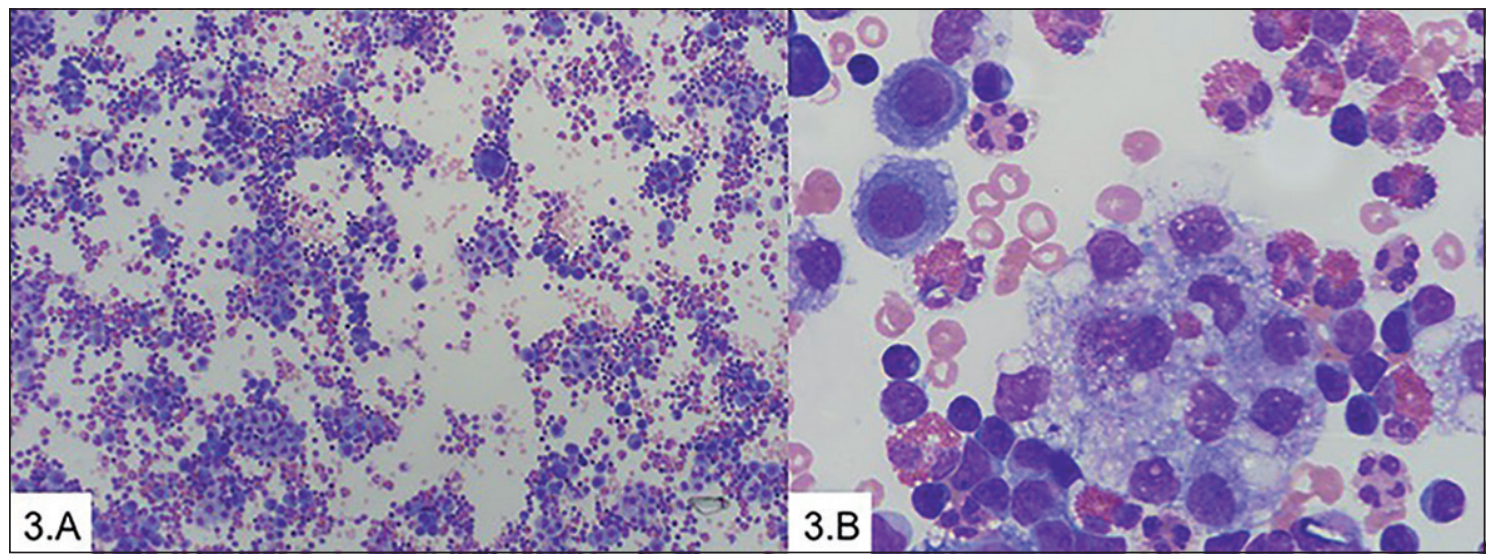

Figura 3. Derrame pleural que muestra abundante compromiso deeosinófilos (células en naranjo); se muestran células mesoteliales (células pequeñas en violeta) y linfocitos (células pequeñas, violeta).

tosoma y Strongyloides), serología para Aspergillus fue negativa. Dado el hallazgo de la neumonía eosinofílica, asociado a al presencia de eosinofilia periférica, en ausencia de causas infecciosas o secundarias a drogas o fármacos, se planteó el diagnóstico de NEC, iniciándose prednisona 1 $\mathrm{mg} / \mathrm{kg}$ peso, obteniendo una disminución de la fiebre al segundo día y disminución de los requerimientos de oxígeno y de la eosinofilia a los 5 días y una resolución de las condensaciones pulmonares y al mes de tratamiento, logrando suspender de manera escalonada los corticoides. Dentro del seguimiento, el paciente presentó dos recurrencias del cuadro respiratorio, de similares características, con buena respuesta a tratamiento con corticoides. Actualmente se encuentra con dosis de mantención de $5 \mathrm{mg}$ al día de prednisona, lo que confirma el diagnóstico de NEC.

\section{Discusión}

La NEC es una patología poco frecuente, de etiología aún desconocida, afectando al sexo femenino en una proporción 2:1 con respecto a los hombres, principalmente en la edad media de la vida ${ }^{1,2}$. Los pacientes que son afectados por una NEC suelen tener antecedentes de asma en $50 \%$ de los casos, aunque también se ha asociado a rinitis alérgica y pólipos nasales ${ }^{3,4}$. En el caso de nuestro paciente, estos antecedentes no estaban presentes. Se ha pesquisado una baja prevalencia, $<10 \%$, de fumadores con esta patología, aunque aún se desconoce si el tabaco ejerce algún efecto protector en el desarrollo de esta enfermedad ${ }^{4}$. En alguna oportunidad se ha descrito el efecto de ciertos medicamentos en el desarrollo de la NEC (antidepresivos, antiinflamatorios, antibióticos, entre otros $)^{5}$. Si bien el uso de levofloxacino ha sido reportado como agente causal de eosinofilia pulmonar ${ }^{5}$, en nuestro caso no parece tener injerencia en el desarrollo del cuadro clínico, dado que el tiempo transcurrido entre su administración y la presencia de síntomas fue de tan sólo una semana.

La NEC suele iniciarse como un proceso pulmonar subagudo, con síntomas de semanas o meses de evolución, pudiendo progresar en ocasiones a la insuficiencia respiratoria grave. Sus síntomas clásicos incluyen tos no productiva, fiebre, disnea leve y baja de peso, que tienden a instalarse en el curso de semanas a meses ${ }^{6}$. Todos estos aspectos coinciden con el cuadro clínico descrito, dado que la sintomatología corresponde a la experimentada por el paciente, en un transcurso de dos semanas. En variadas ocasiones el diagnóstico se plantea tras el fracaso en el tratamiento de una posible neumonía infecciosa, así como suele confundirse con exacerbaciones asmáticas en pacientes con el antecedente previo. En general, la NEC no presenta compromiso extra-respiratorio ${ }^{4}$, como sí ocurre en otras enfermedades pertenecientes a los eosinofilias pulmonares, el diagnóstico diferencial incluye causas tales como la reacción adversa a medicamentos, la neumonía eosinofílica aguda, la aspergilosis broncopulmonar alérgica, causada por el hongo Aspergillus fumigatus; síndrome de 
Löeffler, causado por helmintos y cuyo diagnóstico se logra aislando el parásito; el síndrome hipereosinofílico (SHE) y la vasculitis de Churg-Strauss, entre otros ${ }^{7,8}$. En nuestro caso se descartaron todas las causas de hipereosinofilia secundaria, incluyendo estudio medular para descartar leucemia o trastorno mieloproliferativo como el SHE, causas conocidas de hipereosinofilia pulmonar ${ }^{8}$.

La principal diferencia entre la neumonía eosinofílica aguda y la NEC es la presencia de eosinofilia periférica y en esputo, junto a la elevación de IgE sérica, que se describe en la mayorías de los casos de $\mathrm{NEC}^{9}$. La eosinofilia periférica puede ser variable entre los pacientes, pero requiere un mínimo de $6 \%$ en sangre periférica ${ }^{3}$ o ser mayor a $1 \mathrm{~g} / \mathrm{L}^{4}$. El recuento diferencial promedio de la eosinofilia periférica es de $26 \%$. Si bien no constituye valor diagnóstico, permite monitorizar la respuesta terapéutica, ya que los eosinófilos séricos tienden a normalizarse con el tratamiento $^{10}$. Cuando no existe eosinofilia periférica es obligación documentar eosinofilia pulmonar mediante LBA o biopsia pulmonar ${ }^{3,11,12}$. El LBA y la demostración de eosinofilia en el aspirado del lavado son una prueba de alto valor diagnóstico, de baja morbimortalidad y que ha disminuido la necesidad de biopsia ${ }^{10}$.

Desde el punto de vista histopatológico, se observan abundantes infiltrados eosinofílicos a nivel alveolar e intersticial, con eventual bronquiolitis obliterante asociada y raramente se asocia con vasculitis. La presencia de fibrosis en la biopsia apoya el diagnóstico de NEC, hallazgo presente en nuestro caso. La eosinofilia en el LBA es un hallazgo presente en todos los casos, con un recuento promedio de $58 \%{ }^{13}$.

La radiografía de tórax se caracteriza por mostrar infiltrados alveolares periféricos con compromiso de lóbulos superiores en $50 \%$ de los casos ${ }^{11}$. La imagen "en negativo del edema pulmonar" es infrecuente e inespecífica, observándose en no más de $25 \%$ de los $\operatorname{casos}^{4,11}$. El derrame pleural también es infrecuente, y cuando aparece suele ser asintomático y autolimitado. Excepcionales también son las imágenes nodulares y cavitadas ${ }^{4,14,15}$.

En la TAC de tórax son hallazgos característicos la presencia de focos de condensación, afectando principalmente a regiones periféricas de la zona media y superior del pulmón. El patrón en "vidrio esmerilado" es característico, pero no patagnomónico ${ }^{16}$. Si bien nuestro paciente pre- sentó un derrame pleural evidenciable a la TAC, la distribución de éste no corresponde netamente a lo descrito en la literatura, dado que si bien hubo compromiso de LII y LSI, el compromiso no fue periférico, sino únicamente ubicado en zona lobar inferior izquierda.

Respecto a las pruebas funcionales respiratorias, no existe un patrón característico en la NEC, presentándose tanto patrones restrictivos como obstructivos.

La NEC puede diagnosticarse por una clínica y radiología compatibles, con o sin eosinofilia periférica. Estos hallazgos, junto a los aportados por el LBA, sustentan el diagnóstico y pueden evitar la realización de una biopsia abierta, convirtiéndose así el LBA en una prueba de alta rentabilidad diagnóstica y con una baja morbimortalidad ${ }^{11}$.

Como criterios diagnósticos de NEC se pueden emplear ${ }^{17}$ :

1. Síntomas respiratorios generalmente de más de dos semanas de evolución

2. Eosinofilia en sangre periférica. Eosinofilia en LBA $>40 \%$.

3. Infiltrados pulmonares periféricos.

4. Exclusión de cualquier causa conocida de la enfermedad pulmonar eosinófilica.

Es por esta clasificación que hemos considerado que las manifestaciones clínicas de nuestro paciente corresponderían a una NEC y se ha enfocado como tal su diagnóstico y tratamiento. Si bien existen elementos en la historia clínica que nos abren posibilidades a otros diagnósticos diferenciales, clasificar la patología del paciente como una NEC parece ser lo más apropiado.

El pilar fundamental del tratamiento son los corticoides, con una mejoría rápida de los síntomas en las primeras $72 \mathrm{~h}$ de tratamiento. La ausencia de respuesta clínica a esteroides debe hacer replantear el diagnóstico. Por su parte, la respuesta radiológica completa se logra en las primeras dos semanas en $50 \%$ de los pacientes ${ }^{3}$. Las dosis iniciales eficaces están comprendidas entre $0,5-1 \mathrm{mg} / \mathrm{kg}$ de prednisona al día, pudiendo disminuirse las primeras semanas, hasta lograr una dosis de mantenimiento que fluctúa entre 5-20 mg/día ${ }^{4}$. Se recomienda una duración de tratamiento de al menos seis meses, dada la alta frecuencia de recaídas con la suspensión. Si la enfermedad recidiva al suspender o disminuir la dosis de corticoides, se recomienda mantener el 
tratamiento por al menos un año adicional antes de intentar suspender nuevamente el tratamiento. Existen series de casos que han utilizado corticoides inhalados como coadyuvantes para disminuir las dosis de corticoides sistémicos, pero su utilidad aún no está del todo dilucidada ${ }^{3,4,20}$. Nuestro paciente presentó una mejoría clínica y radiológica favorable al tratamiento con corticoides, dentro del seguimiento presentó dos nuevos eventos de las similares características, lo que es concordante con el diagnóstico de NEC.

En general, el pronóstico de la NEC es bueno, aunque se ha documentado 5\% de los casos que han evolucionado a fibrosis pulmonar ${ }^{19}$. Este hecho, junto a la rápida respuesta clínica y radiológica con el uso de corticoesteroides, resalta la importancia de establecer un diagnóstico y tratamiento precoces, para así evitar las posibles complicaciones asociadas.

\section{Referencias}

1. Carrington CB, Addington WW, Gelf AM, Madoff IM, Marks A, Schwaber JR, et al. Chronic eosinophilic pneumonia. N Engl J Med 1969; 280: 787-98.

2. Mochizuki Y, Kobashi Y, Nakahara Y, Tanaka A, Kawamura T, Sasaki S, Kawanami R. Chronic eosinophilic pneumonia-a follow study of 12 cases. Nihon Kokyuki Gakkai Zasshi 2002; 40 (11): 851-5.

3. Jederlinic PJ, Sicilian L, Gaensler EA. Chronic eosinophilic pneumonia: a report of 19 cases and a review of literature. Medicine 1988; 67(3): 154-62.

4. Marchand E, Reynaud-Gaubert M, Lauque D, Durieu J, Tonnel A-B, Cordier J-F. Idiophatic chronic eosinophilic pneumonia: a clinical and follow-up study of 62 cases. Medicine 1998; 77 (5): 299-312.

5. http://www.pneumotox.com/ [último acceso: 20 de octubre de 2015].

6. Serra J, Montserrat JM, Xaubet A, Picado C, Agustí A. Neumonía eosinófila crónica. Revisión de doce casos. Arch Bronconeumol 1986; 22: 238-42.

7. Pope AL, Davis WB, Allen ED, Christoforidis AJ, Allen JN. Acute eosinophilic pneumonia. A summary of 15 cases and review of the literature. Medicine 1996; 75(6): 334-42.

8. Fernández-Bussy S, Antúnez M, Pires Y, Labarca G.
Endobronchial Involvement in Idiopathic Hypereosinophilic Syndrome. J Bronchology Interv Pulmonol 2015; 22 (4): 329-31.

9. Parra FM, Ferrando MC. Neumonía eosinófila. Caso clínico. Alergol Inmunol Clin 2000; 15: 101-4.

10. Fernández E, Blanquer R, Domínguez A, Herrejón A, Franco J, Palop J, et al. Neumonía eosinófila crónica. Análisis de 4 casos. Arch Bronconeumol 1994; 30: 4147.

11. Gaensler EA, Carrington CB. Peripheral opacities in chronic eosinophilic pneumonia: The photographic negative of pulmonary edema. Am J Roentgenol 1977; 128: 1-13.

12. Marchand E, Etienne-Mastroniani B, Chanez P, Lauque D, Leclerc P. Idiophatic chronic eosinophilic pneumonia and asthma: how do they in each other? Eur Respir J 2003; 7 (22): 8-13.

13. Samman YS, Wali SO, Abdelaal MA, Gangi MT, Krayem AB. Chronic eosinophilic pneumonia presenting with recurrent massive bilateral pleural effusion: case report. Chest 2001; 119 (3): 968-70.

14. Rutgers SR, Schweitzer M. Chronic eosinophilic pneumonia with pleural effusion. Neth J Med 1999; 54 (5): 191-6.

15. Johkoh T, Muller NL, Akira M, Ichikado K, Suga M, Ando M, et al. Eosinophilic lung diseases: Diagnostic accuracy of thin-sections TC in 111 patients. Radiology 2000; 216 (3): 773-80.

16. Martínez Campos T, Ramírez Arroyo V, Arroyo Domingo A, Villafañe Olmedo M. Neumonía eosinófila crónica: a propósito de un caso. Semergen 2011; 37 (6): 321-3.

17. Pérez Arellano JL, Pardo J, Hernández Cabrera M, Carranza C, Angel Moreno A, Muro A. Manejo práctico de una eosinofilia. An Med Int 2004; 21: 244-52.

18. Allen JN, Magro CM, Kings MA, The eosinophilic pneumonias. SeminRespirCrit Care Med 2002; 23 (2): 127-34.

19. Fujimori K, Shimatsu Y, Suzuki E, Arakawa M, Gejyo F. Levofloxacin-induced eosinophilic pneumonia complicated by bronchial asthma. Nihon Kokyuki Gakkai Zasshi 2000; 38 (5): 385-90.

20. Minakuchi M, Niimi A, Matsumoto H, Amitani R, Mishima M. Chronic eosinophilic pneumonia: treatment with inhaled corticosteroids. Respiration 2003; 70 (4): 362-6. 